\title{
Spinal internuncial neurones in progressive encephalomyelitis with rigidity
}

\author{
D. A. H OWE LL, A. J. LEES, A N D P. J . T O G H I L L
}

From the Department of Neuropathology of the Derbyshire Royal Infirmary, Derby, and the Neurological Unit of University College Hospital and the General Hospital, Nottingham

SUMMARY The clinical and pathological features of a fourth patient with progressive encephalomyelitis with rigidity are reported and compared with those previously described. It is suggested that the muscular rigidity, abnormal postures, painful muscular spasms, and myoclonus are a product of excessive and abnormal discharges of alpha motor neurones caused by their release from control by spinal internuncial neurones. A count of neuronal perikarya in the ventral horns confirmed that the disease selectively destroyed small and medium sized neurones, most of which were spinal internuncial neurones. Experimental, clinical, and pathological evidence concerning spinal internuncial neurones is reviewed and found to conform to this theory. The pathogenesis of opsoclonus may be similar.

Kasperek and Zebrowski (1971) described a patient with a complex, progressive, neurological disorder eventually dominated by board-like rigidity of muscles, abnormal postures, and painful muscular spasms. Similar patients were described by Lhermitte et al. (1973) and Whiteley et al. (1976). The postmortem examination in all three patients showed an encephalomyelitis of the spinal cord and brainstem, suggestive of a viral infection, affecting both grey and white matter. The pyramidal tract, and to a lesser extent the lower motor neurones, were spared from destruction by the disease, producing a unique and distinctive appearance. We report a fourth patient with the same disease with a selective destruction of small and intermediate sized spinal neurones, most of which were spinal internuncial neurones. We suggest that the abnormal discharges of the alpha motor neurones causing rigidity, abnormal postures, painful muscle spasms, and myoclonus in our patient were a direct consequence of their release from control by the destroyed spinal internuncial neurones.

\section{Case report}

The patient was a housewife who died at the age

Address for reprint requests: Dr D. A. Howell, Department of Neuropathology, Derbyshire Royal Infirmary, London Road, Derby DE1 2QY.

Accepted 12 March 1979 of 56 years after a complex illness lasting 26 months. A succession of neurological signs and symptoms were superimposed one after another, with only a few brief periods of partial remission or abrupt exacerbation on the progressive downhill course.

Her symptoms began in May 1974, when she developed giddy spells lasting about half an hour. During these spells her head and neck flushed, she sweated profusely, the rest of her body tingled, and her gait was unsteady. Occasionally they were associated with a sense of rotation and diarrhoea. Four months later she complained of brief attacks of severe burning pain in the left side of the neck and head, each lasting 10 seconds or so. By December 1974, her gait was continuously unsteady, and she held her head flexed towards the right side. Stiffness of her fingers made housework difficult.

In February 1975 she was admitted to the General Hospital, Nottingham, after a brief episode of loss of consciousness on rising from bed. This was followed by recurrent attacks of severe vertigo and vomiting, subsiding after four days. She complained of pain in her neck and a stinging feeling all over her body.

On physical examination at that time she was alert and co-operative. There was a flame-shaped haemorrhage in the left fundus. Elevation of the left eye was impaired, and there was a rotary nystagmus on lateral gaze to either side, after the 
vertigo had subsided. The head was drawn to the right and neck movements were limited, painful, and resisted. She had difficulty in relaxing her muscles. There was ataxia of the arms and legs without weakness or wasting of muscles or any loss of sensation in her limbs or trunk. Her gait was ataxic and shuffling. The left triceps and ankle jerks were absent, and the right plantar response was extensor.

Her past health had been good except for stress incontinence and episodes of dysuria after vaginal hysterectomy in 1965. She had mild hypertension (blood pressure $160 / 110 \mathrm{mmHg}$ ).

Investigations included normal full blood count, blood urea and electrolytes, erythrocyte sedimentation rate, liver function tests, blood glucose, chest and skull radiographs, and serum vitamin B12. Serological tests for syphilis and a gamma scan of the brain were also negative. Cerebrospinal fluid (CSF) examination, however, revealed clear, colourless fluid under normal pressure with 36 white cells per $\mathrm{mm}^{3}$ (95\% lymphocytes) and 0.52 $\mathrm{g} / \mathrm{l}$ of protein. A course of adrenocorticotrophic hormone (ACTH) therapy was given with only transient slight improvement. In June 1975 a repeat lumbar puncture yielded CSF with a normal cell count and glucose, and a protein level of $0.81 \mathrm{~g} / \mathrm{l}$. In July 1975 the CSF contained five white cells per $\mathrm{mm}^{3}$ and $0.52 \mathrm{~g} / 1$ of protein. Serological tests for syphilis were negative and the Lange colloidal gold curve was flat. While in the ward she developed burning pains radiating around her chest.

In October 1975 she was transferred to University College Hospital under the care of Dr G. M. Stern for additional opinions about the nature of her illness. At this stage she complained mostly of abnormal movements and postures. She kept her eyes closed because she was distressed by the light, by double vision, and by jerky movements of objects about her. Her arms and legs tingled and were clumsy. She had difficulty in chewing and swallowing. On examination she was mildly depressed but otherwise showed no mental abnormality and gave a lucid and precise account of her illness. Her speech was slurred and the slightest exertion made her breathless. When she opened her eyes there was divergent strabismus with both eyes tending to look downwards. Conjugate movements were otherwise full, though continuously interrupted by vertical or rotary conjugate jerks. It was these conjugate dancing eye movements (opsoclonus) which were associated with the apparent movement of objects about her (oscillopsia). Frequent grimacing and lip smacking movements were present but there were no abnormal movements of the tongue or palate. There was no rigidity of the muscles of the face or jaws. Her head was drawn to the right and slightly back by rigid neck muscles, and attempts to correct the posture were resisted involuntarily by increased painful muscular spasm. Severe plastic rigidity was present in the right arm and left wrist. There was painful restriction of movement of the right shoulder. The abdominal muscles were rigid and felt hard. The leg muscles were rigid, causing the legs to lie extended and slightly internally rotated at the hips with inversion of the ankles. The left arm shook continuously with a two or three per second coarse flapping tremor. A milder intermittent but similar tremor affected the muscles rotating the left hip more than the right one. Shock-like contractions of muscles of the left arm, particularly the biceps brachii, and of the left leg occurred spontaneously, and the brief muscular spasms spread a little to neighbouring muscles before subsiding slowly over several seconds. They were very painful. These painful myoclonic jerks or spasms were easily provoked by examination, making the assessment of muscle power difficult, but it was thought that there was only a little generalised weakness. She was thin, but the only definite wasting was in the small muscles of the hands. The left leg was ataxic in the heel-kneeshin tests. All tendon reflexes were absent, and the plantar responses were extensor. Vibration sense and joint position sense were normal, but there was patchy sensory impairment to pinprick over the limbs and trunk, and two-point discrimination was impaired in the hands.

Routine haematological and biochemical investigations were normal, as was the urinary 4-hydroxy3-methoxymandelic acid (VMA). Two urinary 5hydroxy-indoleacetic acid (5IAA) estimations were within the normal range of 15 to $75 \mu \mathrm{mol} / 24 \mathrm{hr}$, but the third was $150 \mu \mathrm{mol} / 24 \mathrm{hr}$. The Mantoux test was 1 in 1000 and the tests for rheumatoid and antinuclear factor were negative. The electrocardiogram, electroencephalogram, skull radiographs, gamma scan of brain, vertebral angiogram, and computerised axial tomograms of the brain were all normal. Nerve conduction studies showed normal motor nerve conduction velocities and a slightly reduced median nerve sensory action potential.

A course of dexamethasone was given without benefit, but diazepam gave slight relief from the painful spasms.

The patient returned to Nottingham in November 1975 and remained in hospital until she died 
in June 1976. In January the tremor, the orofacial dyskinesias, and the painful spasms subsided, perhaps in response to treatment with chlorpromazine, but in March the painful spasms in the left arm and leg became almost continuous. They were provoked by noise or any emotional distress. Intravenous diazepam gave some relief. Rigidity became more severe and was not relieved by tetrabenazine or benzhexol. The extensor posture of the legs changed to one of flexion at the hip and knees, her muscles wasted, and she became cachectic before she died.

\section{NECROPSY AND HISTOLOGY REPORT}

At postmortem examination (Dr S. Variend) she was an emaciated woman (weight $32 \mathrm{~kg}$, height $1450 \mathrm{~mm}$ ). There was severe generalised muscls: wasting and flexion contraction of the hips and knees. The bronchi contained pus and the lungs were oedematous. The bladder mucosa was patchily haemorrhagic. The brain weighed $1330 \mathrm{~g}$. Neither the brain nor the spinal cord showed any convincing naked eye abnormality then or when they were sectioned, after fixation in formalin. Blocks were embedded in paraffin and sections were stained by a variety of methods.

Sections of muscle were available, and these showed a moderate neurogenic atrophy.

Sections of peripheral nerve showed no abnormality though lymphocytic cuffing of a solitary vessel was found in the fifth cranial nerve distal to the junction between the central and peripheral type of myelination.

Sections of a spinal root ganglion showed that $10-15 \%$ of the spinal root ganglion cells were replaced by empty capsule cell clusters, the residual nodules of Nageotte. This loss of cells was larger than usual but of uncertain significance.

There was a patchy scattering of lymphocytes in the subarachnoid space and around some of the small vessels penetrating the white and grey matter of the spinal cord. Occasionally this was accompanied by perivascular proteinous lakes. Vessels showed collagenous thickening, as did the arachnoid mater. Some subintimal oedema of vessels was also present. Small vessels were prominent in the grey matter, many capillaries showing thin collagenous sheaths, and there was a general appearance of engorgement of the grey matter. Clusters of microglia were uncommon in the dorsal horns and intermediate grey matter and rare in the ventral horns. Some ventral horn cells showed chromatolysis and most contained much lipofuscin.

Increase in astrocytes and astrocytic fibres were prominent in preparations stained by Holzer's method, and fairly uniform throughout the grey matter of the spinal cord, though the gliosis was a little less dense in the ventral horns of the grey matter than in the intermediate zone, and much less in the substantia gelatinosa. Loss of neuronal cell bodies was much more selective. Small and medium sized neurones had virtually disappeared from most of the grey matter, but there was only a moderate loss of small neurones from the substantia gelatinosa in the tip of the dorsal horns and from the ventral horns. The larger neurones of the nucleus dorsalis of Clarke were depleted but the medium sized neurones of the intermediolateral nucleus in the lateral horns appeared unaffected by the disease. The large neurones appeared to be preserved in the ventral horns (Fig. 1).

There was widespread loss of myelin in the spinal cord. It was accompanied by moderate astrocytic gliosis in the Holzer preparations, but no lymphocytic infiltration beyond the perivascular spaces. In sections stained by Holmes' method the greatest demyelination was accompanied by loss of axons and fenestration of the tissue. In less demyelinated regions the axons appeared normal. The well-stained myelin of the spared corticospinal tracts, and the spinal motor neurones stood out in dramatic contrast (Fig. 2). The dorsal columns were partially spared, particularly in the thoracic and lumbar segments.

Sections at six levels through the medulla oblongata showed a similar lymphocytic perivascular and meningeal infiltration, with a little meningeal and vascular collagenous thickening, especially in the closed medulla. Many neurones had disappeared from the inferior olives, particularly the ventral folds, to be replaced by astrocytes. Microglial stars and clusters of lymphocytes were frequent in the dorsal folds of the inferior olives (Fig. 3). The number of astrocytes was increased throughout the medulla oblongata, and sections stained by the Holzer method showed an astrocytic gliosis, not obviously accompanied by a loss of neurones.

In sections of the medulla stained for myelin the intact corticospinal pathway stood out against the general loss of myelin. However myelinated hypoglossal nerve fibres appeared normal and there was little demyelination of the fibres of the medial lemniscus or the tractus solitarius. The loss of myelin was less intense rostrally, though astrocytic gliosis was present.

In the pons, the basis pontis showed no disease, but there was a little, perivascular, lymphocytic cuffing in the pontine tegmentum and clusters of microglial cells in one of the abducens nuclei. 


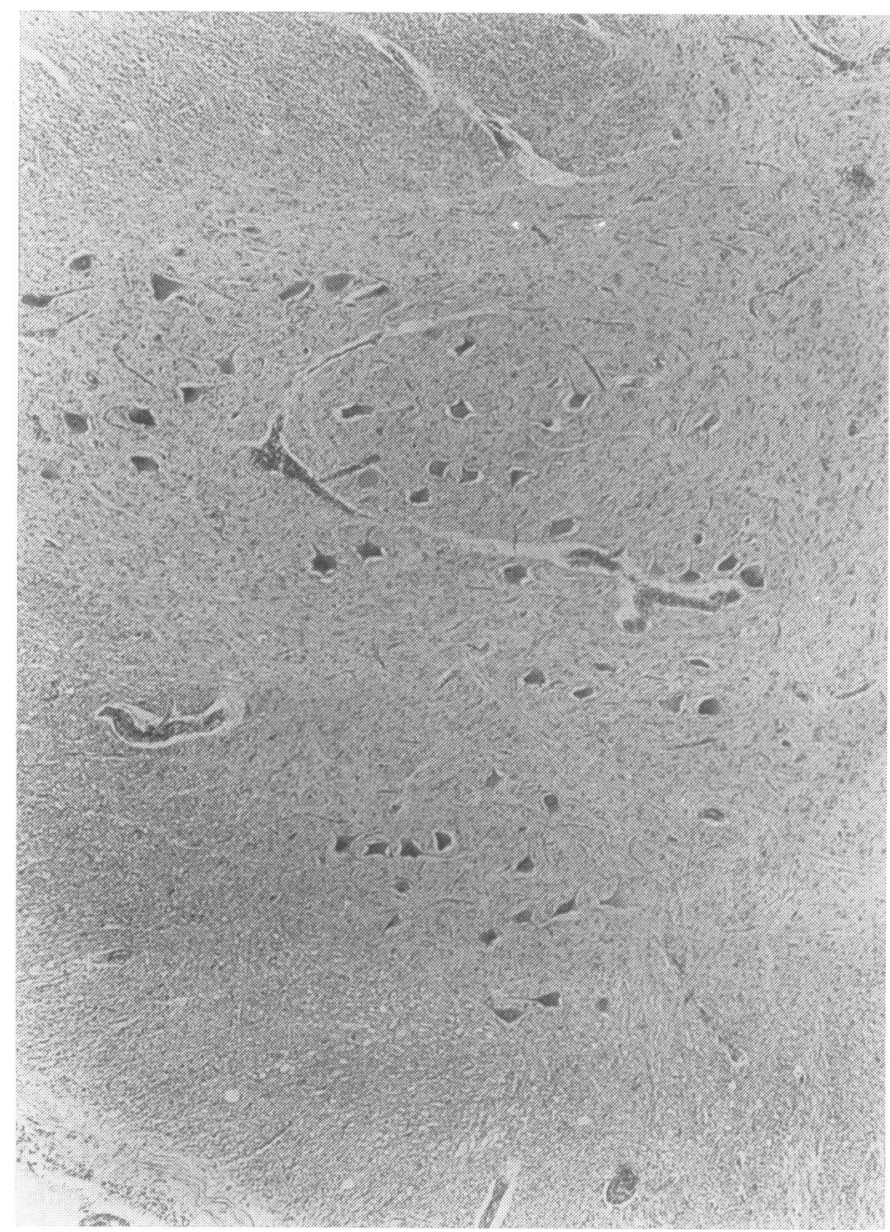

Fig. 1 Anterior horn of fifth cervical segment stained by Nissl's method, original magnification $\times 52$. Small and intermediate sized nerve cells are absent but the large cells are well preserved. Perivascular cuffing with lymphocytes and a microglial star are present.

The myelinated fibres of the tegmentum were pale in comparison with the basis pontis, particularly the medial lemniscus. The astrocytic gliosis in sections stained by Holzer's method was not dense, and no obvious neuronal loss was seen.

The cerebellar cortex showed no abnormality other than a little agonal degeneration of granule cells. The central white matter, however, was a little demyelinated and contained vessels surrounded by cuffs of lymphocytes. Many of the neurones of the ventral and lateral portion of the dentate nucleus were missing and replaced by a growth of fibrous astrocytes. Neuronophagic figures or microglial clusters were present here. This was much more marked in one dentate nucleus than in the other.

The midbrain showed a little perivascular cuffing around vessels entering the posterior perforated space. Though no loss of neurones was recognised there was a dense astrocytic gliosis, in the section stained by Holzer's method, throughout the whole of the periaqueductal grey matter and a similar less intense gliosis in the tectum. The lateral lemniscus was poorly myelinated and contained an excessive number of astrocytic fibres. The sections stained for myelin showed no other abnormality.

Sections through the cerebral hemisphere showed no significant abnormality. Neurones of the cortex, basal ganglia, and diencephalon appeared normal. A little pallor of the myelin of the centrum semiovale was associated with acute swelling of the interfascicular oligodendroglia and, therefore, considered to be agonal. Rare trivial perivascular cuffs with a few lymphocytes were seen.

COUNT OF NEURONAL PERIKARYA IN THE VENTRAL HORNS

We decided to test whether the apparent loss of small and intermediate neuronal cell bodies in the 

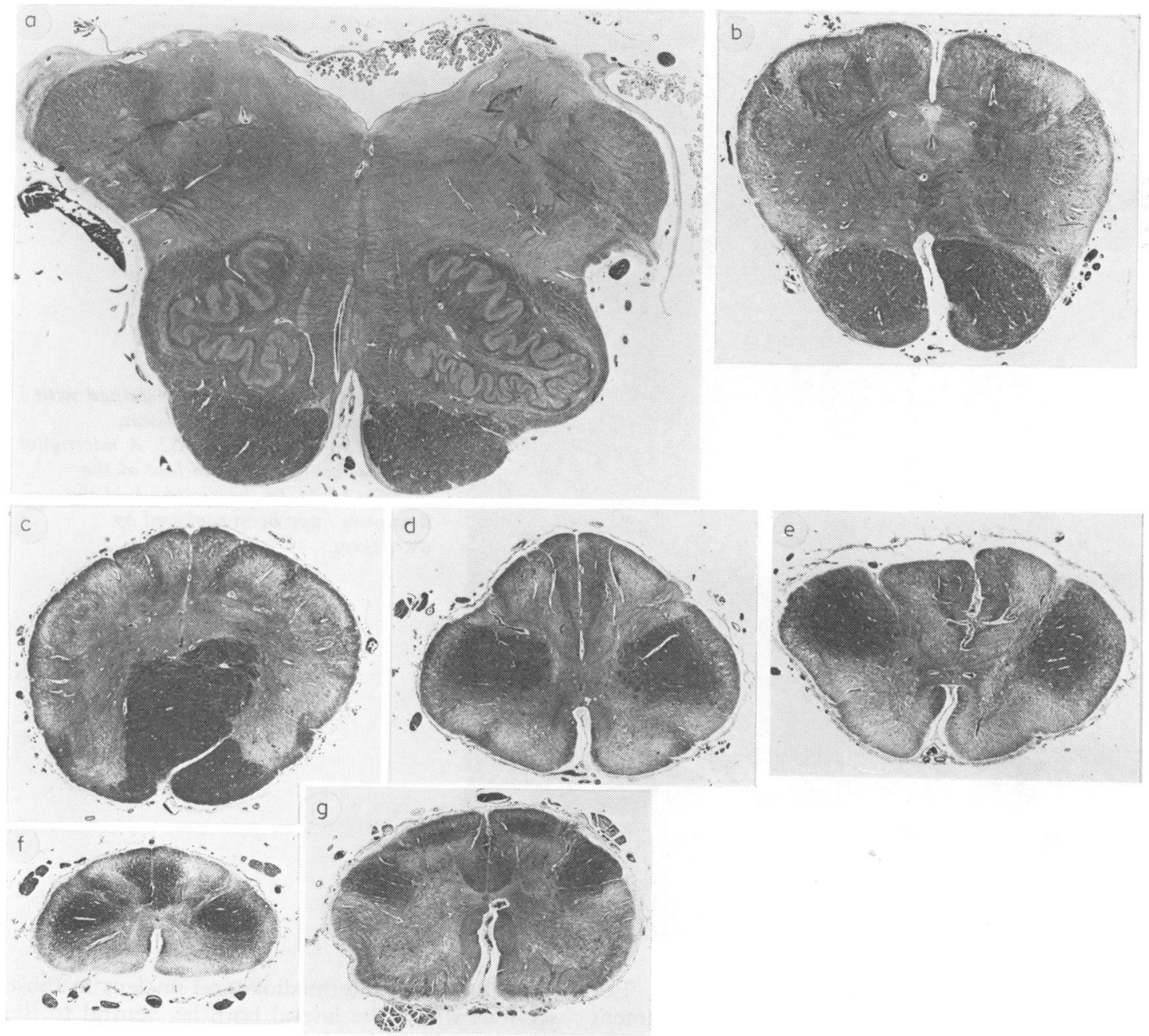

Fig. 2 Transverse sections through the medulla oblongata and spinal cord stained by the Loyez method for myelin. Sparing of the corticospinal tract and lower motor neurones from the widespread demyelination is shown.

grey matter of the spinal cord was real by counting and measuring them. The apparent loss was recognised throughout the spinal cord from upper cervical to sacral segments, and was more obvious in the base of the dorsal horns than in the ventral horns of grey matter. But for practical reasons we decided to count and measure cells in the ventral horns of the eighth thoracic segment. A count of all the cells in the grey matter might have been more difficult to interpret because of the obvious sparing of many small neurones of the substantia gelatinosa, intermediate neurones of the lateral horns, and the large neurones of the nucleus dor- salis, none of which were thought relevant to the disorder of movement and muscle tone. In the ventral horns even small neurones have cytoplasm full of Nissl and, usually, lipofuchsin granules and a large round nucleus with a prominent nucleolus, easily distinguished from other cells (Fig. 4). The nuclei of some active astrocytes were similar, but if any cytoplasm was visible at all it contained no granules. In the dorsal horns the faint granularity of the cytoplasm of small neurones was much more difficult to distinguish from the hyaline cytoplasm of swollen astrocytes, and counting neurones would be more difficult. The ventral horns could 


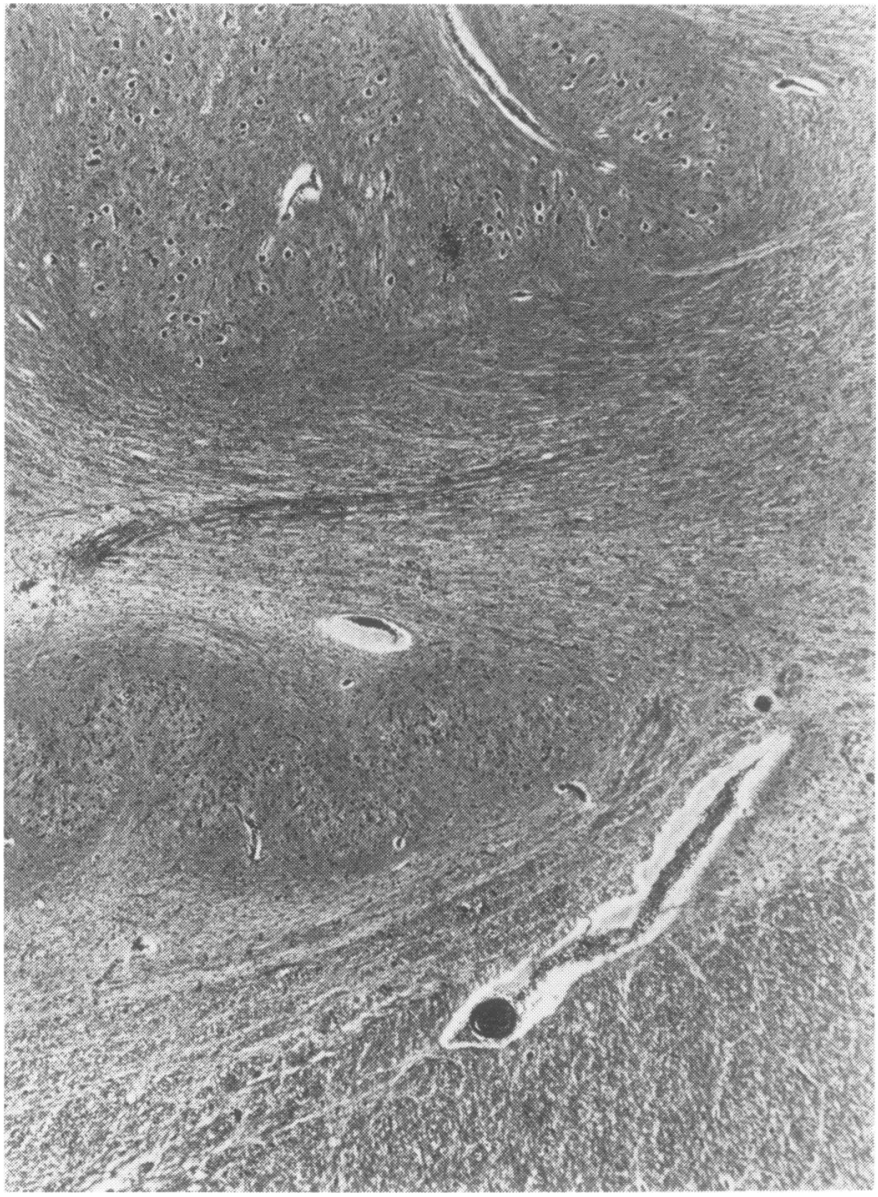

Fig. 3 Medulla oblongata stained with haematoxylin and Van Giesson, original magnification $\times 52$. A microglial star is seen in the dorsal fold of the inferior olive. In the ventral fold the neurones have been replaced by astrocytes.

be defined easily for counting purposes. For clinical correlation a lumbar or cervical segment would have been preferable, but it was very difficult to match in control specimens. The thoracic spinal cord does not vary so much from millimetre to millimetre. The eighth thoracic segment innervated abdominal muscles described as rigid in the case records.

Serial paraffin sections were cut on a Leitz base sledge microtome with the thickness control set at $15 \mu \mathrm{m}$, from the eighth thoracic segment of the spinal cord of the patient and of a control patient of the same age and sex who died after rupture of an intracranial aneurysm. The blocks were processed together and stained by Lapham's method, chosen because it allowed the nucleolus to be distinguished by colour from large round Nissl granules overlying the nucleus. The ventral horns were defined as all the grey matter lying ventral to the ventral white commissure, exclud- ing cells of the intermediolateral nucleus in those sections where the lateral horn lay ventral to the ventral white commissure. The maximum diameter of the neuronal cell bodies was measured in the section containing the nucleolus using a Reichert "Visopan" microscope. The cells were arranged in classes containing all cells with a maximum cell body diameter falling within limits separated by $5 \mu \mathrm{m}$. Two hundred cells were counted in 95 serial sections from the patient and in the control patient in only $16 \frac{1}{2}$ serial sections. The numbers in each class were plotted in a histogram from the patient and from the control patient after multiplying by a factor to give the number of cells in the same length of spinal cord (Fig. 5).

As a further check on the validity of the count, neurones were counted and measured in exactly the same way from the same spinal cord segment of three other control patients. These results are shown in the second histogram (Fig. 6) after 


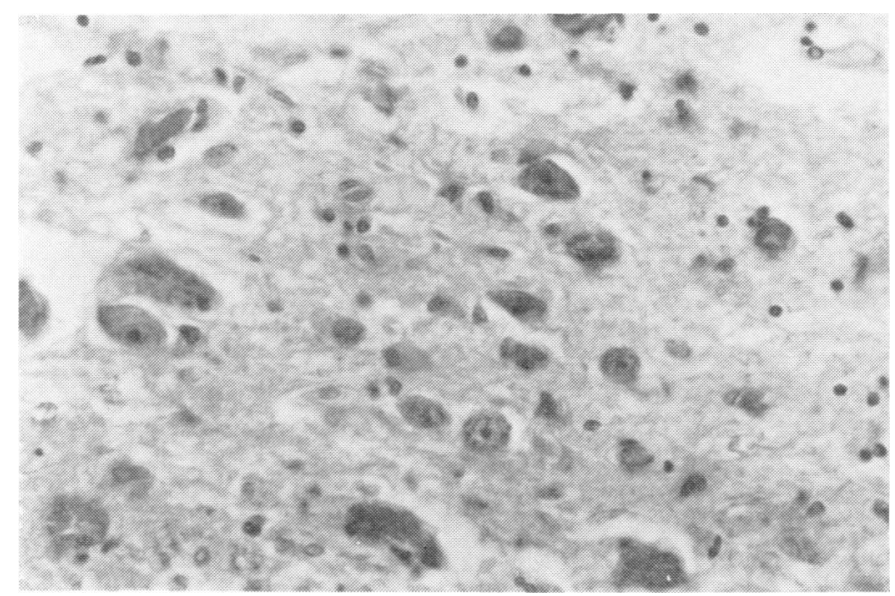

Fig. 4 Transverse section through T8 segment from the patient, original magnification $\times 650$, stained by Lapham's method. It shows a unique group of neuronal cell bodies and swollen astrocytes near the dorsal border of the ventral horn. Four neurones with granular cytoplasm have nucleoli in focus. They measure $12,14,20$, and $23 \mu \mathrm{m}$ in diameter respectively. Three swollen astrocytes show small nucleoli. The hyaline cytoplasm distinguishes them from the granular neurones.

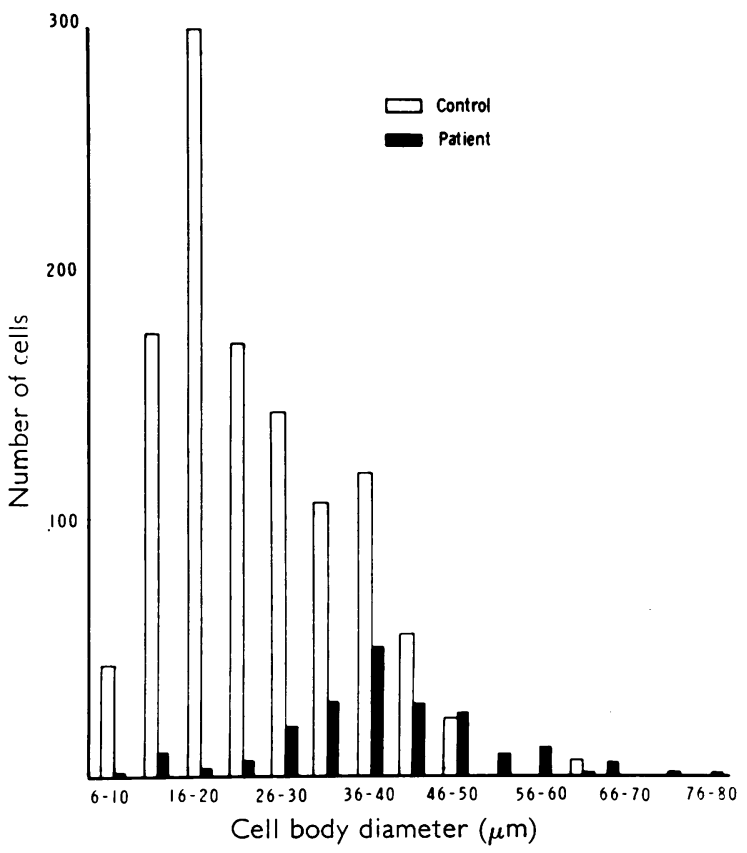

Fig. 5 Histogram showing numbers of neuronal perikarya of different diameters in the ventral horns in the same length of the eighth thoracic segment of the patient and a control patient of the same age and sex.

multiplying by a factor to give the number of neurones in the same length of spinal cord as in the patient. In the histogram, $A$ is the count in the original control patient, $B$ is the result of counting 200 neurones in $17 \frac{1}{2}$ sections from a man aged 37 years who died of pneumonia while convalescing from acute inflammatory peripheral neuropathy. Recovery was incomplete and some chromatolysis was present. Section $C$ is the result of counting 200 neurones in $14 \frac{1}{2}$ sections in a woman aged 28 years with von Recklinghausen's disease who died from an anaplastic astrocytoma of the corpus callosum. Column D is the result of counting 200 neurones in 17 sections from a woman aged 50 years who died from a pulmonary embolus eight weeks after the onset of acute multiple sclerosis of the spinal cord which rendered her quadriplegic. The eighth thoracic segment showed a plaque of multiple sclerosis affecting both ventral horns.

While it is impossible to deny that some neurones may have been overlooked, it is thought that the important source of error arises from counting neurones twice. It was essential to be on 


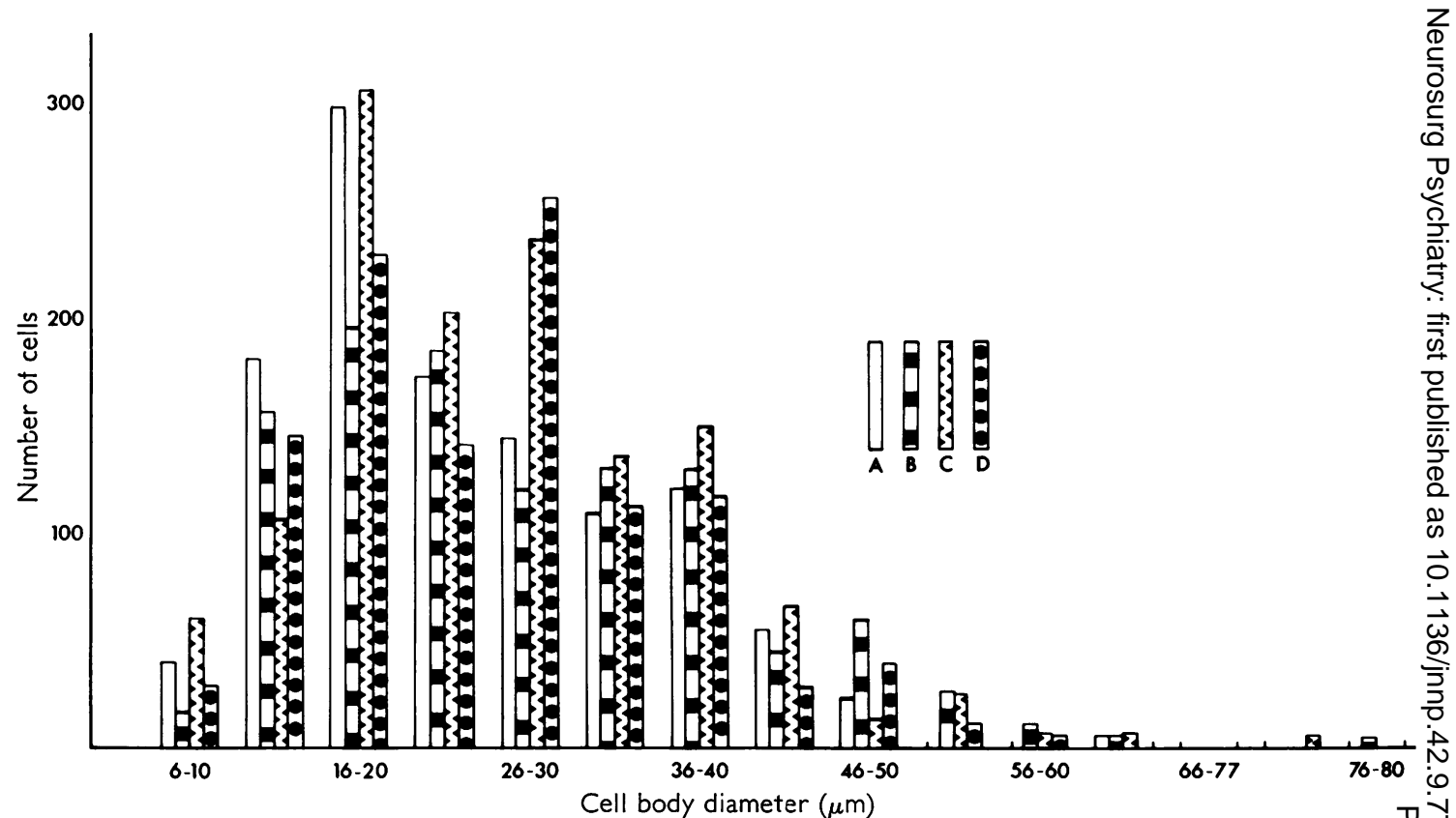

Cell body diameter $(\mu \mathrm{m})$

Fig. 6 Histogram showing numbers of neuronal perikarya of different diameter in the ventral horns in the same length of the eighth thoracic segment of four control patients. A-a woman aged 56 yr with a ruptured intracranial aneurysm; $B$-a man aged $37 \mathrm{yr}$ with acute inflammatory peripheral neuropathy; $C-a$ woman aged $28 \mathrm{yr}$ with an anaplastic astrocytoma of the corpus callosum; D-a woman aged $50 \mathrm{yr}$ with multiple sclerosis.

constant guard against the human error of counting the same neurone twice in the well-populated ventral horns of the four control cords, though there was no difficulty in the patient with an average of only one neurone per ventral horn. In addition to any human counting error, nucleoli may be divided in two, and the same cell may be counted in two sections. This error may be avoided if there is only a fragment of a nucleolus in one section or on the very rare occasions when a full sized nucleolus is recognised in the same cell in two sections. It exaggerates the number of large cells with large nucleoli more than small cells. As paraffin sections vary slightly in thickness the factor used to give the number of neurones in the same length of spinal cord is a little inaccurate.

Nonetheless the patient and the four control subjects showed only a little difference in the number of large neurones. In the cat $90 \%$ of these are thought to be alpha motor neurones (Romanes, 1964) and in man the proportion of alpha motor neurones with a cell body diameter above $33 \mu \mathrm{m}$ in celloidin sections of the lumbar cord is even higher (Kawamura et al., 1977b). This diameter is approximately equivalent to $30 \mu \mathrm{m}$ in paraffin sections. The moderate neurogenic atrophy available sections of muscle indicates that the apparent, more than two-fold loss of these celfs $\vec{\theta}$ was real. The numbers of large neurones above 6 $30 \mu \mathrm{m}$ in diameter in the same length of spinal 0 cord were: patient, 142; control A, 317; control B, 411; control C, 401, and control D, 319. The loss of small and intermediate sized neurones measuring less than $30 \mu \mathrm{m}$ in diameter in the $\mathbb{\otimes}$ patient was between eighteen and twenty-four-fold. $\overrightarrow{\vec{t}}$ The numbers of small and intermediate sized neurones in the same length of spinal cord were: patient, 38; control A, 834; control B, 672; control 㕿 C, 910 , and control D, 588. A loss of such magnitude is, in our opinion, far too great to be accounted for by counting errors. Among the intermediate sized neurones lie the gamma motor neurones, but in man there are only between half and a third as many gamma as alpha motor neurones (Kawamura et al., 1977a). Nearly all the remaining small and intermediate sized neurones are thought to be internuncial neurones. We be- $\rightarrow$ lieve that the results of the count confirm that the disease selectively destroyed small and inter- N mediate sized neurones in the ventral horns, though large neurones were not unscathed. 


\section{Discussion}

OTHER PATIENTS WITH PROGRESSIVE

ENCEPHALOMYELITIS WITH RIGIDITY

An indolent encephalomyelitis involving the grey and white matter of the spinal cord and brainstem with sparing of the upper and most of the lower motor neurones produces a distinctive pathological picture. As far as we know, it has only been described in our patient and in those of Kasperek and Zebrowski (1971), Lhermitte et al. (1973), and Whiteley et al. (1976). These four patients may be considered to be suffering from one disease though there were some differences.

The inflammatory cellular infiltrate was relatively scanty in our patient. It reached up to the hippocampus in the patient of Kasperek and Zebrowski (1971) and to the globus pallidus in the patient of Lhermitte et al. (1973). The inferior olive and dentate nucleus was more severely damaged in our patient. There was some variation in the pattern of demyelination in the spinal cord. It is inevitable that minor variations in intensity and distribution of disease in such an eloquent part of the nervous system must be associated with major clinical differences, though muscular rigidity, abnormal postures, and painful muscle spasms were features common to all four patients.

Kasperek and Zebrowski (1971) described the illness in a woman who was 49 years old when she died 17 months after the onset. The illness began with pain in the sacrum radiating down the legs, followed by burning pains and other painful dysaesthesiae in all the limbs. She developed a little sensory impairment in a glove and stocking distribution, slight generalised muscular weakness, and loss of tendon reflexes. Muscular rigidity was first noted as a stiffness in the arms six months after the onset. It progressed so that the arms were held in a bizarre posture and movement was limited. The muscles of the arms and shoulder girdle were board-like on palpation. Frequent painful spasms involved the shoulder girdle and the legs, the left leg being extended in a spasm. They were provoked by almost any trivial external stimulus such as a gentle touch, slight passive movement, or a question asked in a soft voice. They were associated with profuse sweating and screaming in agony. Electromyographic studies showed persistent normal motor unit activity, enhanced in agonists and antagonists during spasms. The stiffness spread to involve the face. Shortly before she died the spasms ceased to affect the legs which became hypotonic. Treatment with diazepam gave some relief and was associated with a partial remission for a few months during which she was able to sit in a chair and to walk with assistance, though she was bedridden before treatment. During her illness she developed diplopia and eventually a convergent squint with almost constant deviation of the eyes to the right. Some months before her death she became deaf.

Lhermitte et al. (1973) described the illness in a man who was aged 73 years when he died, 39 months after the onset. It began with stiffness in the legs and a sensory ataxia chiefly in the arms. The legs were permanently held in rigid extension 14 months later when there was no muscle weakness. The knees and ankle jerks were brisk and the plantar responses extensor. The rigidity spread to involve the trunk, face, and masseters, but not the arms. Eighteen months after the onset painful muscular spasms appeared, lasting 15 seconds to several minutes, differing only in minor detail from those described by Kasperek and Zebrowski (1971). They were relieved a little by treatment with diazepam and also with baclofen. The arms, which were not rigid, became hypotonic and weak with muscle wasting and fasciculation. The upper limb tendon reflexes were lost. During the illness he developed diplopia with bilateral sixth nerve palsies, changing slowly into an almost complete external ophthalmoplegia with a convergent squint. A loss of ventral horn cells in the cervical cord in the histological sections explained the hypotonia and wasting.

Whiteley et al. (1976) described the illness in a woman aged 62 years when she died, 13 months after the onset. Rigidity was present at the beginning and progressed in the trunk and limbs so that eventually no voluntary movement was possible and all muscle groups felt hard. Postures were maintained against resistance and there was no clasp knife phenomenon or cogwheel element on passively stretching the muscles. Attempts to manipulate the limbs provoked intense, painful, muscular spasms, beginning in the limb examined and slowly spreading to all the muscles of the limbs and trunk. The spasms were easily provoked by almost any stimulus, as in the other two patients, and were accompanied by sweating. The patient suffered from dysuria progressing to complete incontinence of urine and faeces. She showed a coarse tremor in the hands and shaking of the legs early in the illness. Except for slurred speech there was no involvement of the cranial nerves, and sensation was unimpaired in the trunk or limbs. There was no muscular wasting and tendon reflexes were brisk. Treatment with a variety of drugs including diazepam and baclofen had no effect on her rigidity.

Our patient showed features in common with 
the other patients as well as differences. The illness began with bizarre giddy spells containing elements which appeared again as more persistent symptoms. She suffered from painful dysaesthesiae and patchy sensory impairment like the patient of Kasperek and Zebrowski (1971). She developed a cerebellar ataxia early in the illness, while the patient of Lhermitte et al. (1973) suffered from a sensory ataxia. Three patients exhibited different bizarre disturbances of ocular movement, and only the case described by Whiteley et al. (1976) had normal eye movements throughout. Only the patient described by Lhermitte et al. showed profound muscle weakness and wasting but all lost some ventral horn cells. Cranial nerve functions were spared, except for slurred speech, in the case of Whiteley et al., who also showed no sensory loss. She complained of dysuria leading to complete incontinence. Both the latter patient and ours showed a coarse tremor. Only Kasperek and Zebrowski's patient became deaf. Opsoclonus, oscillopsia, and grimacing and lip smacking movement were not seen in the others.

With the exception of the hypotonia in the arms of the patient of Lhermitte et al., the distribution of the rigidity was much the same, but it was much more intense round the shoulders in Kasperek and Zebrowski's patient and in the neck in our patient. The posture was related to the distribution and intensity of the rigidity. The other three patients showed painful muscular spasms spreading into all the rigid muscles. The painful involuntary resistance to correcting the torticollis in our patient was much more localised, and the painful prolonged myoclonic jerks never spread to all the muscles.

\section{SPINAL INTERNUNCIAL NEURONES}

A selective destruction of spinal internuncial neurones remarkably similar to that seen in our patient has been reported by Gelfan and Tarlov (1963) in dogs with hind limb rigidity induced by transient ischaemia of the spinal cord. They occluded the descending thoracic aorta for 40-50 minutes (Gelfan and Tarlov, 1959). They illustrated a pair of symmetrical infarcts in the grey matter of the lumbar spinal cord separated by the grey commissure, stretching from the centre of the dorsal horns into the centre of the ventral horns and presumably stretching through the lumbar and sacral spinal cord. Counts of myelinated fibres in the ventral root of the seventh lumbar segment showed no loss in two rigid dogs, suggesting complete sparing of the alpha and gamma motor neurones in these animals. Counts of neurones in this segment in rigid and normal dogs showed that $80 \%$ of the internuncial neurones were lost. Some destruction of motor neurones was found in animals with a greater loss of internuncial neurones than this.

The hind limbs of these animals showed an abnormal extensor posture and muscular rigidity $\frac{\rho}{5}$ so that the animal could be made to stand, though the legs were paralysed and apparently anaesthetic. ? Monosynaptic tendon reflexes were exaggerated, $\overrightarrow{\vec{F}}$ but flexion reflexes, which travel through a poly- $)$ synaptic pathway involving internuncial neurones, were lost. During the first five days after the tem- $\frac{O}{\bar{N}}$ porary clamping of the aorta the rigidity was $\frac{D}{\vec{D}}$

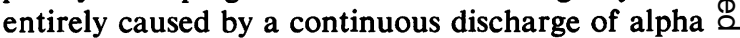
motor neurones to extensor and flexor muscles क alike. With a wealth of electrophysiological $\vec{\circ}$ studies they demonstrated that internuncial neuronal function was lost and also showed that $\vec{\omega}$ monosynaptic responses activated a larger pro- $S$ portion of the available alpha motor neurone pool $\frac{5}{3}$ than in control animals. This form of rigidity was? maintained after sectioning of the dorsal nerve $\stackrel{\vec{N}}{0}$ roots and transection of the thoracic spinal cord, quite unlike spasticity or extrapyramidal rigidity Muramaya and Smith (1965) produced a similat 응 rigidity in cats and also showed that polysynapt reflex responses were diminished or absent. $\cong \vec{~}$

Isolation from, or release from, control by spina $\mathbb{0}$ inhibitory neurones may cause the abnormal be $\mathbb{\mathbb { D }}$ haviour of alpha motor neurones in tetanus an strychnine poisoning. Tetanus toxin and strychnide. have long been known to have similar pharmac $\vec{\omega}$ logical effects on postsynaptic inhibition in the spinal cord (Brooks et al., 1957). It has been shown that glycine is the probable transmitter substance released by spinal internuncial neurones involved in postsynaptic inhibition, and that strychnine acts by limiting the action of glycine upon subsynaptic $\frac{\circ}{\Phi}$

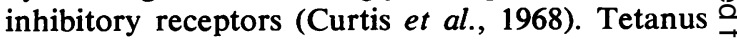
toxin blocks the inhibitory process by reducing the $\overline{\overrightarrow{0}}$ amount of glycine released from inhibitory ter- 3 minals (Curtis and DeGroat, 1968). It also reduces the amount of gamma aminobutyric acid (GABA) released at the terminals of other inhibitory neurones in the brain and spinal cord (Curtis et $\overline{0}$ al., 1973).

In addition to abnormal rigidity and postures, patients with strychnine poisoning or tetanus show abnormal movements not reported in Gelfan and

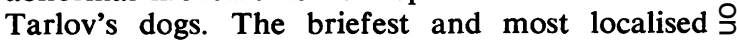
form is the myoclonic jerk of a part of a muscle. $\frac{D}{2}$ The contraction of a muscle may be prolonged into a painful spasm subsiding only after a few $N$ minutes. It may spread to neighbouring muscles including antagonists. It may even involve all the 
muscles of the face, trunk, and limbs in the strychnine convulsions. Such movements occur spontaneously or may be provoked by emotional or sensory stimuli. In strychnine poisoning the symptoms may be relieved by treatment with diazepam (Hardin and Griggs, 1971).

Similar muscular rigidity and abnormal posture associated with a loss of spinal internuncial neurones has been described in patients with subacute necrotic myelitis and with brain trauma to the cervical spinal cord by Penry et al. (1960). A patient with abnormal posture and rigidity of the arms associated with a cervical spinal astrocytoma was reported by these authors and in more detail by Rushworth et al. (1961). The rigidity was also attributed to isolation of the alpha motor neurones from the control of spinal internuncial neurones and they described continuous activity of the motor units in electromyograms of the rigid muscles. They also reported spontaneous sudden bursts of increased motor unit activity occurring in both agonist and antagonist muscles persisting synchronously for several minutes, associated with painful spasms of increased rigidity of sudden onset and slow decline. This could be provoked by stretching a rigid muscle.

Myoclonus may occur in disease localised to the spinal cord. Penfield and Jasper (1954) described a patient with continuous myoclonic jerks of certain muscles of the abdomen and back innervated by the lower thoracic spinal cord. During a laminectomy they found this part of the spinal cord infiltrated by a glioma. Garcin et al. (1968) have described a patient with rhythmic myoclonus in muscles of an arm associated with a cervical cord astrocytoma. In the experimental encephalomyelitis of cats infected with Newcastle disease virus, the animals showed continuous myoclonus which persisted in muscles innervated by a part of the spinal cord isolated from the rest of the nervous system by transection of the spinal cord and cutting dorsal nerve roots (Luttrell et al. 1959). The disease destroyed neurones chiefly in the ventral horns and in the intermediate zone of spinal grey matter. It is reasonable to infer that many alpha motor neurones must have survived as there would have been no myoclonus without them and that internuncial neurones were destroyed. Hopkins and Michael (1974) have published electrophysiological evidence that myoclonus originated in the spinal cord in a patient with probable viral encephalomyelitis. In the histological examination of the third patient described by Campbell and Garland (1956) with subacute myoclonic spinal neuronitis, the inflammation was almost confined to the spinal cord. Neuronal destruction was greater in the dorsal than in the ventral horns. The illness began with stiffness and myoclonic jerks in the legs which evolved into severe painful muscular spasms accompanied by drenching sweats.

Swanson et al. (1962) concluded that myoclonus resulted from an abnormal and excessive discharge of aggregates of internuncial neurones or motor neurones. This may be caused by directly enhanced excitability, removal of some inhibitory mechanism, or both. In the rigid dogs described by Gelfan and Tarlov (1959) they concluded that the abnormal and excessive discharge of the alpha motor neurones causing the muscular rigidity was in turn caused by their isolation from the controlling influences of spinal internuncial neurones. In strychnine poisoning the tetanus and alpha motor neurones are only isolated from the control of certain inhibitory internuncial neurones, and their abnormal and excessive discharge is both continuous and paroxysmal. Myoclonus and painful muscular spasms are added to the muscular rigidity and abnormal postures. In disease of the spinal cord it is probable that the pattern of dysfunction of internuncial neurones may vary, and patients with a loss of function of only some of these neurones will show a combination of muscular rigidity, abnormal postures, muscular spasms and myoclonus, varying from case to case.

In their classification of myoclonus, Swanson et al. (1962) include opsoclonus as a segmental form confined to conjugate eye movements, but often associated with widespread myoclonus as in our patient. They include a report of a patient with viral encephalomyelitis who showed opsoclonus and myoclonus of the trunk and limbs recovering in about a month. Similar cases have been reported by Barringer et al. (1968) and McLean (1970). There are only a few reports of the pathology in patients with opsoclonus. Yakovlev (1942) described a patient dying after a long progressive illness in whom muscular rigidity and myoclonus of the limbs, face and eye movements were prominent symptoms, reminiscent of our patient. There was atrophy of the brainstem and Hallervorden-Spatz type of pathological change in the pallidum, substantia nigra and dentate nucleus. Cogan (1954) reported a patient with diffuse encephalitis in the diencephalon, brainstem, and cerebellum. The distribution of disease was similar in the patient with subacute cerebellar degeneration associated with carcinoma reported by Ross and Zeman (1967), but in the patient with the same condition reported by Ellenberger et al. 
(1968) disease was confined to the cerebellum. Opsoclonus is a rare symptom of cerebellar disease, and the close association with segmental spinal myoclonus makes it difficult to believe that myoclonus of ocular movements is very different in pathogenesis. It is difficult to recognise a loss of large neurones in the brainstem nuclei unless it is fairly gross or they are arranged in a folded ribbon as in the dentate and inferior olivary nuclei. We have not been able to convince ourselves that there is a loss of small neurones in the brainstem, though the increase in astrocyte fibres indicates active disease at some time. Nor have we been capable of excluding a small to moderate loss by comparison with anatomical atlases or normal control sections.

\section{SIMILARITY TO ENCEPHALITIS LETHARGICA}

Whiteley et al. (1976) described a second patient with encephalomyelitis whom they thought might have an accelerated form of the disease. The illness was characterised by aching in the back and legs, weakness of the legs, and attacks of rigidity and jerking in the limbs. It was fatal in three weeks. The demyelination with sparing of the corticospinal tracts, so characteristic of progressive encephalomyelitis with rigidity, was not present, but the illness was perhaps too brief for this change to develop. The substantia nigra was partially destroyed by the disease which was similar to the spinal form of encephalitis lethargica (Ellis, 1920). It was also similar to the cases of subacute myoclonic spinal neuronitis described by Campbell and Garland (1956). Chronic cases were also described during the epidemic of encephalitis lethargica which were very similar to progressive encephalomyelitis with rigidity (Whiteley et al., 1976). It is also possible that a variety of viruses may produce this particular pattern of pathological change. Myoclonus, though not necessarily segmental spinal myoclonus, may be encountered in almost any form of proven or suspected viral infection of the central nervous system including poliomyelitis (Swanson et al., 1962).

\section{SIMILARITY TO STIFF MAN SYNDROME}

Kasperek and Zebrowski (1971) reported their patient as suffering from the stiff man syndrome caused by encephalitis. Gordon et al. (1967) have laid down strict criteria for the diagnosis of stiff man syndrome, and Whiteley et al. (1976) found only 35 patients in the literature conforming to their description. Nearly half of these were reported from the Mayo Clinic, including the original patients described by Moersch and Wolt- man (1956). Adults of both sexes are afflicted. The onset is usually gradual, and the condition becomes static after a variable period of progression. It may improve, but does not recover. The stiffness consists of a fluctuating rigidity of the trunk, limbs, and sometimes the face, so severe as to draw the head down between the shoulders and to deform the spine. The gait is affected, and a patient may fall "like a wooden man." The muscles feel hard and on electromyography show continuous motor unit activity which is only arrested by drugs blocking the myoneural junction, nerve blocks, or general anaesthesia, indicating an excessive abnormal discharge of motor neurones. Myoclonus is unusual but most patients have painful muscle spasms spontaneously or provoked by stimuli such as a fright or sudden movement. They may be so powerful that they break the neck of the femur. A third of the patients show an unidentified reducing substance in the urine. The brain has been examined several times and the spinal cord twice, without revealing any significant abnormalities. The condition may respond dramatically to treatment with diazepam, and this has enabled bedridden patients to return to fulp activity. Gordon et al. (1967) rejected patients̊ whose stiffness might be attributed to muscle pathology and patients with disease of the centrab nervous system which might cause spasticity or extrapyramidal rigidity. It is clear that the patients accepted by Gordon et al. (1967) are rather different from those with progressive en cephalomyelitis with rigidity, and they can be distinguished by their long survival and the absence of other evidence of encephalomyelitis. It is our contention that there are a number of diseases causing a syndrome of muscular rigidity, abnormal postures, painful muscular spasms, and myoclonus, including strychnine poisoning and tetanus as well as gliomas of the spinal cord, encephalomyelitis, and the one or more diseases which may underly the stiff man syndrome. The excessive and abnormal discharge of alpha motor neurones in all these conditions is caused by their release from control by spinal internuncial neurones.

We thank the Trent Regional Health Authority Research fund for purchasing the Reichart "Visopan" microscope. We are also grateful to Professor B. E. Tomlinson, Professor T. A. Sears, and Professor A. T. Birmingham for advice, to $\mathrm{Mr}$ D. M. Roberts-Jones for much technical assistance, and to $\mathrm{Mr} \mathrm{W}$. Brackenberry for the photographs. 


\section{References}

Baringer, J. R., Sweeney, V. P., and Winkler, G. F. (1968). An acute syndrome of ocular oscillations and truncal myoclonus. Brain, 91, 437-480.

Brooks, V. B., Curtis, D. R., and Eccles, J. C. (1957). The action of tetanus toxin on inhibition of motor neurones. Journal of Physiology, 135, 655-672.

Campbell, A. M. G., and Garland, H. (1956). Subacute myoclonic spinal neuronitis. Journal of Neurology, Neurosurgery, and Psychiatry, 19, 268-274.

Cogan, D. (1954). Ocular dysmetria: flutter-like oscillations of the eyes and opsoclonus. Archives of Ophthalmology, 51, 318-335.

Curtis, D. R., and DeGroat, W. C. (1968). Tetanus toxin and spinal inhibition. Brain Research, 10, 208212.

Curtis, D. R., Felix, D., Game, C. J. A., and McCulloch, R. M. (1973). Tetanus toxin and synaptic release of GABA. Brain Research, 51, 358-362.

Curtis, D. R., Hosli, L., Johnston, G. A. R., and Johnston, I. H. (1968). The hyperpolarisation of spinal motoneurones by glycine and related amino acids. Experimental Brain Research, 5, 235-258.

Ellenberger, C., Campa, J. F., and Netsky, M. G. (1968). Opsoclonus and parenchymatous degeneration of the cerebellum. Neurology (Minneapolis), 18, 1041-1046.

Ellis, A. W. (1920). The myoclonic form of acute epidemic encephalitis. Lancet, 2, 114-116.

Garcin, R., Rondot, P., and Guiot, G. (1968). Rhythmic myoclonus of the right arm as the presenting symptom of a cervical cord glioma. Brain, 91, 75-84.

Gelfan, S., and Tarlov, S. M. (1959). Interneurones and rigidity of spinal origin. Journal of Physiology, 146, 594-617.

Gelfan, S., and Tarlov, I. M. (1963). Altered neurone population in L7 segment of dogs with experimental hind limb rigidity. American Journal of Physiology, 205, 606-616.

Gordon, E. E., Januszko, D. M., and Kaufman, L. (1967). A critical survey of stiff man syndrome. American Journal of Medicine, 42, 582-599.

Hardin, J. A., and Griggs, R. C. (1971). Diazepam in a case of strychnine poisoning. Lancet, 2, 372-373.

Hopkins, A. P., and Michael, W. F. (1974). Spinal myoclonus. Journal of Neurology, Neurosurgery, and Psychiatry, 37, 1112-1115.

Kasperek, S., and Zebrowski, S. (1971). Stiff man syndrome and encephalomyelitis. Archives of Neurology (Chicago), 24, 22-30.

Kawamura, Y., Okazaki, H., O'Brien, P. C., and Dyck, P. J. (1977a). Lumbar motoneurons of man. I. Number and diameter histogram of alpha and gamma axons of ventral root. Journal of Neuropathology and Experimental Neurology, 36, 853859.

Kawamura, Y., Okazaki, H., O'Brien, P. C., and Dyck, P. J. (1977b). Lumbar motoneurons of man.
II. Number and diameter distribution of large and intermediate diameter cytons in "motoneuron collumns" of spinal cord of man. Journal of Neuropathology and Experimental Neurology, 36, 861870.

Lhermitte, F., Chain, F., Escourolle, R., Chedru, F., Guilleminault, C. and Franconal, M. (1973). Un nouveau cas de contracture tetaniforme distinct du "stiff man syndrome". Revue Neurologique, 128, 3-21.

Luttrell, C. N., Bang, F. B., and Luxenberg, K. (1959). Newcastle disease encephalomyelitis in cats. $A r$ chives of Neurology and Psychiatry (Chicago), 81, 285-291.

McLean, D. R. (1970). Polymyoclonia with opsoclonus. Neurology (Minneapolis), 20, 508-512.

Moersh, F. P., and Woltman, H. W. (1956). Progressive fluctuating muscular rigidity and spasm ("stiff man" syndrome): report of a case and some observations in 13 other cases. Proceedings of the Staff Meetings of the Mayo Clinic, 31, 421-427.

Muravama, S., and Smith, C. M. (1965). Rigidity of hind limbs of cats produced by occlusion of spinal cord blood supply. Neurology (Minneapolis), 15, 565-577.

Penfield, W., and Jasper, H. (1954). Epilepsy and the Functional Anatomy of the Human Brain, chapter 2, page 36. Little, Brown and Co: Boston.

Penry, J. K., Hoefnagel, D., Van Den Noort, S., and Denny-Brown, D. (1960). Muscle spasm and abnormal postures resulting from damage to interneurones in spinal cord. Archives of Neurology (Chicago), 3, 500-512.

Romanes, G. F. (1964). The motor pools of the spinal cord. In Progress in Brain Research, volume 11, pp. 93-116. Edited by J. C. Eccles and J. P. Schade. Elsevier: Amsterdam.

Ross, A. T., and Zeman, W. (1967). Opsoclonus, occult carcinoma and chemical pathology in the dentate nuclei. Archives of Neurology (Chicago), 17, 546-551.

Rushworth, G., Lishman, W. A., Hughes, T., and Oppenheimer, D. R. (1961). Intense rigidity of the arms due to isolation of motoneurones by a spinal tumour. Journal of Neurology, Neurosurgery, and Psychiatry, 24, 132-142.

Swanson, P. D., Luttrell, C. N., and Magladery, J .W. (1962). Myoclonus - a report of 67 cases and review of the literature. Medicine (Baltimore), 41, 339-356.

Whiteley, A. M., Swash, M., and Urich, H. (1976). Progressive encephalomyelitis with rigidity. Its relation to subacute myoclonic spinal neuronitis and to the stiff man syndrome. Brain, 99, 27-42.

Yakovlev, P. I. (1942). A case of myoclonus epilepsy with atrophy of the brain stem and HallervordenSpatz type of pathological change in the pallidum, substantia nigra and Dentate nucleus. Transactions of the American Neurological Association, 68, 95100. 